\title{
Disease Pattern and Outcome of Neonates at Special Newborn Care Unit of Pokhara Academy of Health Science, Nepal
}

\author{
Bastola RC ${ }^{1}$, Shrestha SK ${ }^{1}$, Ghimire $\mathbf{J J}^{1}$, Gurung $\mathbf{R}^{1}$, Sigdel YR ${ }^{1}$ \\ ${ }^{1}$ Pokhara Academy of Health Sciences
}

Received: 5-May-2017; Accepted: 30-June-2017

\begin{abstract}
Aims: This study was conducted to identify the causes and the outcome of neonates admitted to SNCU of Western Regional Hospital.

Methods: A retrospective study was conducted at SNCU of western Regional Hospital, a referral tertiary centre of Western Region of Nepal from March 2016 to March 2017.

Results: Total of 1144 neonates were admitted in NICU comprised of 562 (49.12.\%) neonatal sepsis, 164 (14.33\%) neonatal hyperbilirubinemia, $120(10.48 \%)$ birth asphyxia, $109(9.52 \%)$ premature, $92(8.04 \%)$ meconium aspiration syndrome, 96 (8.39\%) congenital anomalies and $14(1.22 \%)$ necrotizing enterocolitis. Out of neonatal sepsis, $20.62 \%$ were early onset and $28.49 \%$ late onset conditions. Among birth asphyxia, $102(85.00 \%)$ were of HIE I\& II and $18(15 \%)$ of HIE III. Overall mortality observed was $3.32 \%$. Those who required mechanical ventilation had higher mortality $(87.50 \%)$ than the cases that were kept for CPAP (14.81\%).
\end{abstract}

Conclusions: Neonatal sepsis, birth asphyxia, prematurity and neonatal hyperbilirubinemia were major causes for admission in SNCU.

Keywords: birth asphyxia, hyperbilirubinemia, mortality, neonatal, prematurity, sepsis

DOI: 10.3126/njog.v12i2.19956

\section{INTRODUCTION}

The neonatal mortality of Nepal as per Nepal Demographic and Health Survery (NDHS) 2016 data is 21 per 1,000 live births. ${ }^{1}$ Neonatal period (0-28 days of life) is the most vulnerable period of life due to different life threatening diseases, which in most cases are preventable. ${ }^{2}$ Almost $50 \%$ of child deaths in our country occur in the neonatal period. ${ }^{1}$ Though considerable improvement in the survival of newborn in developed countries has been made but the mortality rate is still very high in the developing countries. $^{3}$ One of the target of Sustainable Development Goals is to reduce the number of deaths among children by the year 2030, and to achieve this goal a substantial reduction in neonatal deaths will be required especially in the developing countries. ${ }^{4}$ In the developed countries, the main cause of morbidity and mortality in the neonatal period is congenital abnormalities which are mostly non-preventable, but

\section{CORRESPONDENCE}

Dr Ramchandra Bastola

Consultant Pediatrician,

Department of Paediatrics,

Pokhara Academy of Health Sciences, Pokhara ,Nepal

Email: dr_rc@yahoo.com in the developing countries like Nepal, the common causes such as infections,prematurity, jaundice, birth asphyxia and meconium aspiration syndrome predominates for neonatal mortality. ${ }^{5}$ The neonatal disease pattern is a sensitive indicator of availability, utilization and effectiveness of mother and child health services in the community. Of the 130 million neonates born every year. Globally, about 4 million die in the first 4 weeks of life. ${ }^{6}$ Most of the neonatal deaths $(99 \%)$ occur in the lower to middle income countries and half of them occur at home. The risk of a newborn dying is 24 per 1,000 live births in the first week of life, 3 per 1,000 per week during the rest of the first month, and 0.12 per 1,000 per week after the first year of life. ${ }^{7}$ Most of the causes we encounter in NICU of neonatal mortality and morbidity are preventable. ${ }^{8-9}$ The pattern of neonatal disease varies from place to place..$^{10}$ The major causes of neonatal deaths globally were estimated to be infections (35\%), preterm births (28\%) and asphyxia (23\%). ${ }^{11}$ A study previously done in Dharan, Nepal shows prematurity as the leading cause of hospital admission (23.8\%) followed by birth asphyxia (20.2\%), Early onset neonatal sepsis (16.3\%), Late 
onset neonatal sepsis (16.3\%), meconium aspiration syndrome $(8.3 \%)$, neonatal hyperbilirubinemia $(4.7 \%)$, cardiac anomalies $(2.7 \%)$, congenital anomalies $(2.7 \%)$, Necrotizing enterocolitis $(2.4 \%)$, Transient tachypnoea of newborn (1.3\%) and with mortality after using Mechanical ventilator of $20.2 \%$ but saved life of $64.2 \%$ those who were in MV. ${ }^{12}$ All these diseases can be prevented by good obstetric and pediatric care. The aim of this study was to identify the common causes for admission to the NICU and its outcome.

\section{METHODS}

A retrospective study conducted at the Special Newborn Care Unit (SNCU) of Western Regional Hospital which is also now known as Pokhara Academy of Health Sciences (PAHS), Pokhara, Nepal from $30^{\text {th }}$ March 2016 to $30^{\text {th }}$ March 2017. A total number of 1144 neonates admitted to SNCU were included in the study. Our SNCU is a tertiary care centre of having facilities of mechanical ventilation, CPAP, radiant warmers and double surface LED phototherapy which are needed for the care of sick neonates. The data of all the neonatal admissions were documented and analyzed by age, gender, place of delivery, cause of admission, syndrome (MAS) (Table 1). birth weight and final outcome. Diagnosis was based mainly on clinical with specific laboratory or radiological findings. Sepsis and meningitis were diagnosed on clinical grounds along with C-reactive protein (CRP), complete blood count (CBC), micro ESR, positive blood culture and cerebrospinal fluid (CSF) examination. Congenital heart disease was diagnosed mainly on clinical basis, chest X-Ray and then confirmed by echocardiography. Birth asphyxia was diagnosed clinically and hypoxic ischemic encephalopathy (HIE) by Sarnat and Sarnat staging. Diagnosis of prematurity was mainly clinical using new Ballard scoring or based on WHO definition for prematurity (live born neonates delivered before 37 weeks from 1st day of last menstrual period). Low birth weight was defined as when birth weight was less than $2500 \mathrm{~g}$. Neonatal jaundice was first diagnosed by Kramer staging and then assessing through serum bilirubin level.

\section{RESULTS}

Total 1144 neonates were admitted in NICU during our study period; $619(54.10 \%)$ were males and 525 (45.89\%) females. The most common cause of admission was neonatal sepsis followed by neonatal hyperbilirubinemia, birth asphyxia, prematurity, congenital anomalies and meconium aspiration

Table1: Common presentation at neonatal unit

\begin{tabular}{|lllll|}
\hline Major Diseases & Disease Classification & Number & Individual Percent (\%) & Total Percent (\%) \\
\hline \multirow{3}{*}{ Early Onset Neonatal Sepsis } & Sepsis & 122 & $51.69 \%$ & \\
& Pneumonia & 69 & $29.23 \%$ & $236(20.62 \%)$ \\
& Meningitis & 45 & $19.06 \%$ & \\
Late Onset Neonatal Sepsis & Meningitis & 153 & $46.93 \%$ & $326(28.49 \%)$ \\
& Pneumonia & 64 & $19.63 \%$ & $164(14.33 \%)$ \\
Neonatal Hyperbilirubinemia & Sepsis & 109 & $33.43 \%$ & $120(10.48 \%)$ \\
Birth Asphyxia & HIE grade I, II & 164 & & $109(9.52 \%)$ \\
Prematurity & HIE grade III & 18 & $15.00 \%$ & \\
Meconium Aspiration Syndrome & Moderate & 109 & & $92(8.04 \%)$ \\
Congenital anamolies & Severe & 78 & $87.74 \%$ & $96(8.39 \%)$ \\
NEC & & 14 & $15.21 \%$ & $14(1.22 \%)$ \\
Total & & 14 & & $14(100 \%)$ \\
\hline
\end{tabular}

Those who required continuous positive airway pressure (CPAP) had better improvement but neonates who required mechanical ventilation had very poor outcome (Table-2). 
Table 2: Outcome of neonates

\begin{tabular}{|llll|}
\hline & Improved & Expired & Total \\
\hline CPAP used & $138(83.95 \%)$ & $24(14.81 \%)$ & $162(14.16 \%)$ \\
Mechanical Ventilator used & $2(12.50 \%)$ & $14(87.50 \%)$ & $16(1.39 \%)$ \\
\hline
\end{tabular}

\section{DISCUSSION}

Western regional hospital (WRH) or Pokhara Academy of Health Sciences (PAHS) being a tertiary-care center, get many neonates in advanced stage of the disease or complicated by their illness from whole western region of Nepal accounting more than 5 million population. Males predominate as study subjects, which is similar to other studies. ${ }^{12}$ This may be because of the fact that males get more attention on part of caregivers and brought to the hospital for seeking health services in our country. Only neonatal sepsis accounted for $49.11 \%$ cases requiring admission in our SNCU. A slightly lower incidence $(41.3 \%)$ has been reported by Jan et al. ${ }^{11}$ and lower incidence (6.4-10.5\%) by other authors in their studies. ${ }^{13,14}$ The variation in occurrence of sepsis depends upon the health practices being followed in the community and awareness of health professionals and parents providing the neonatal care. Since late onset accounted more for NNS than early onset in our study, predisposing factors like late attention to the health facilities, poor referral system during antennal, intrapartum and postnatal period are equally important and should be taken into consideration in its prevention.

Neonatal hyperbilirubinemia is the cause of admissions in $14.33 \%$ of neonates. Much higher incidence $(36.2-54 \%)$ has been reported in other studies. ${ }^{13,15,19}$ The SNCU admissions for neonatal hyperbilirubinemia is mainly done for double surface LED phototherapy. Early detection, proper bilirubin monitoring and effective double surface LED phototherapy made almost no need of exchange phototherapy in our study.

Birth asphyxia (10.48\%) accounted for the third most common cause of admissions to our SNCU and mostly (85.00\%) of HIE stage II and $15.00 \%$ of stage III. The incidence is almost similar to that of Butt et $\mathrm{al}^{15}$ and much lower than reported from South Africa. ${ }^{17}$ Thus, occurrence of high incidence of birth asphyxia and mostly in severe stages indicate the level of neonatal care existing in our heath sector especially in the community. So, adequate attention should be paid in giving training for health workers who can effectively reduce the incidence as well as its severity and decrease the load of SNCU admissions at tertiary care level.

Prematurity was found to be the next common cause $(9.52 \%)$ for admission. This is slightly low to the incidence reported from South Africa. ${ }^{17}$ But a much higher incidence (34.6- 48.2\%) has been reported from the other neighboring country Pakistan. ${ }^{13,16}$ Preterm neonates really require very close attention and advanced care from the experienced staff nurse and neonatologist because of their inherent handicaps and complications. Their higher incidence in developing world is a major concern, so preventive aspects should be taken into consideration beside establishment of NICU or SNCU in different regions of the country.

Meconium aspiration syndrome was found in $8.04 \%$ of cases, which also require urgent attention at the time of birth in the form of tracheal suctioning if the baby is depressed. Similar incidence has been reported $(8.30 \%)$ in a different study done in eastern Nepal, Dharan by Shah et al. ${ }^{12}$ However, this can be performed efficiently in inborn neonates where trained resident staff attends the delivery. Prakash and $\mathrm{Das}^{18}$ reported the incidence of $3.67 \%$ in their cases.

Congenital anomalies were found in 96(8.39\%) of neonatal admission. Congenital malformations are not rare in our country and most commonly affected system were ear, eye, face and neck anomalies and syndromic baby followed by digestive, genitourinary, musculoskeletal, CVS, and skin in descending order of frequency. An Indian study ${ }^{20}$ revealed first ranking for CNS followed by musculoskeletal and then CVS. Necrotising enterocolitis (NEC) were found only in 14 cases, i.e. $1.22 \%$ of total admission.

Overall mortality observed was $3.32 \%$. Those who required mechanical ventilation had higher mortality $(87.50 \%)$ than the cases that were kept for CPAP (14.81\%). Thus, it appears that the need of CPAP in SNCU is very crucial in countries like us where the neonatal mortality is still very high and due to financial strains, the government couldn't establish adequate NICU or SNCU. Higher mortality of neonates kept in Mechanical ventilation shows itself 
a risk factor for mortality. Jan et $\mathrm{al}^{12}$ also found lower mortality $(8.3 \%)$ in their audit report. The mortality depends upon the stage of the disease and facilities available in a particular NICU or SNCU. Moreover, attempt should be made to keep it as low as possible.

\section{REFERENCES}

1. Ministry of Health, New ERA ICF. 2017. Nepal Demographic and Health Survey 2016: Key Indicators. Kathmandu, Nepal: Ministry of Health, Nepal; 2017.

2. Rahim F, Mohammad AJ, Iqbal H. Patterns and outcome of admissions to neonatal unit of Khyber Teaching Hospital, Peshawar. Pak J Med Sci. 2007:23:249-53.

3. Ng PC. Diagnostic members of infection in neonates. Arch Dis Child Fetal Neonatal Ed. 2004;89: F229-35.

4. National Planning Commission. Sustainable Development Goals, 2016-2030, National (Preliminary) Report. Government of Nepal, National Planning Commission, Kathmandu, Nepal; 2015

5. Khinchi YR, Kumar A, Yadav S. Profile of neonatal sepsis. J Coll Med Sci Nepal. 2010;6(2):1-6.

6. Jehan I, Harris H, Salat S, Zeb A. Moben N, Pasha O, et al Neonatal mortality: risk factors and causes: a prospective population based cohort study in Pakistan. Bull WHO. 2009;87:130-8

7. Aurangzeb B, Hameed A. Neonatal sepsis in hospital born babies: bacterial isolates and antibiotic susceptibility patterns. J Coll Physicians Surg. 2003;13:629-32.

8. Gurubacharya SM, Gurubacharya RL. An overview of neonatal admissions at College of Medical Sciences. J Nepal Paediatr Soc. 2007;27(2):73-4.

9. Parkash J, Das N.Pattern of admission to neonatal unit. J Coll Physician Surg Pak. 2005; 15:34

10. Abbasi KA. Neonatal disease profile in Larkana before and after establishment of neonatal ward. J Pak Med Assoc. 1995;45:235-6

\section{CONCLUSIONS}

Thus, it appears that neonatal sepsis, birth asphyxia, prematurity and neonatal hyper bilirubinemia are the major cause of SNCU admissions at our centre. CPAP used in Neonates has good outcome and prevents many neonates from death. Need for ventilation adversely affected the outcome. This can be reduced by improving antenatal care of pregnant women, timely interventions and of high risk pregnancies to tertiary care center by an experience obstetrician and neonatologist.

11. Jan AZ,Ahmad S, Zahid AB. Clinical audit of admission pattern and its outcome in a neonatal ICU. Gomal J Med Sci. 2013;11:31-6.

12. Shah GS, Yadav S, Thapa A, Shah L. Clinical profile and outcome of neonates admitted to neonatal intensive care unit (NICU) at a Tertiary Care Centre in Eastern Nepal. J Nepal Paediatr Soc. 2013;33(3):177-81

13. Nahar J, Zabeen B, Akhter S, Azad K, Nahar N. Neonatal morbidity and mortality pattern in the special care baby unit of Birdem. Ibrahim Med Coll J. 2007;1:4

14. Ogunlesi TA. Ogunfowora Ob, Adekanmi AF, Feluga MB Runsewe TI. MMO gundeyi, Neonatal mortality at Olabisi Onabanjor University Teaching Hospital, Sagamu. Niger J Pediatr. 2006;33:40-6

15. Butt NA, Malik A, Kazi MY. Pattern of neonatal admissions in tertiary care hospital. Pak J Med Health Sci. 2010;4:436-8.

16. Seyal T, Husanain F, Anwar A. Audit of neonatal morbidity and Mortality at Neonatal unit of Sir Gangaram Hospital Lahore. Annal King Edward Med Coll. 2011;1:9-13

17. Hoque M, Haque M, Haaq S, Islam R. Causes of neonatal admission and deaths at a rural hospital in Kwa Zulu-Nall, South Africa. South Afr J Epidemiol Infect. 2011;26-9.

18. Narayan R. A study of pattern of admission and outcome in a neonatal intensive care unit at high altitude. Sri Lanka J Child Health. 2012;41:79-81

19. Sharma P, Kaur P, Aggarwal A. Staphylococcus aureus- the predominant pathogen in the neonatal ICU of a tertiary care hospital in amritsar, India. Clin Diagn Res. 2013;7(1):66-9.

20. Parmar DA, Rathod DSP, Patel DSV, Patel DSM. A study of congenital anomalies in newborn. NJIRM. 2010;1:13-7. 